\title{
Buttiauxella gaviniae
}

National Cancer Institute

\section{Source}

National Cancer Institute. Buttiauxella gaviniae. NCI Thesaurus. Code C122254.

A species of facultatively anaerobic, Gram-negative, rod-shaped bacteria in the family Enterobacteriaceae. B. gaviniae is positive for arginine dihydrolase, 5-ketogluconate, and palatinose reactions and negative for ornithine decarboxylase and raffinose reactions. 